\title{
Magnetic field control of the Franck-Condon coupling of few-electron quantum states
}

\author{
P. L. Stiller, ${ }^{1}$ A. Dirnaichner, ${ }^{1}$ D. R. Schmid, ${ }^{1}$ and A. K. Hüttel ${ }^{1,2, *}$ \\ ${ }^{1}$ Institute for Experimental and Applied Physics, University of Regensburg, 93040 Regensburg, Germany \\ ${ }^{2}$ Low Temperature Laboratory, Department of Applied Physics, Aalto University, P.O. Box 15100, FI-00076 Aalto, Finland
}

(Received 6 December 2018; revised 18 August 2020; accepted 20 August 2020; published 9 September 2020)

\begin{abstract}
Suspended carbon nanotubes display at cryogenic temperatures a distinct interaction between the quantized longitudinal vibration of the macromolecule and its embedded quantum dot, visible via Franck-Condon conductance sidebands in transport spectroscopy. We present data on such sidebands at known absolute number $N_{\mathrm{el}}=1$ and $N_{\mathrm{el}}=2$ of conduction band electrons and, consequently, well-defined electronic ground and excited states in a clean nanotube device. The interaction evolves only at a finite axial magnetic field and displays a distinct magnetic-field dependence of the Franck-Condon coupling parameter, different for different electronic base states and indicating a valley dependence. Reshaping of the electronic wave function by the magnetic field is discussed as a possible cause of our observations; its impact is demonstrated in a model calculation reproducing the field-dependent coupling.
\end{abstract}

DOI: 10.1103/PhysRevB.102.115408

\section{INTRODUCTION}

Vibrational degrees of freedom, typically approximated at small deflection as harmonic oscillators, contribute in many ways to the fundamental properties of matter. The FranckCondon principle $[1,2]$ relates vibrational wave functions in molecular physics to the intensity envelope of vibrational sidebands in optical spectra. This principle, where an electronic transition is assumed to be instantaneous compared to the slow motion of the nuclei, also becomes directly visible in electronic low-temperature transport spectroscopy of single (macro)molecules [3-12]. An experimental system where such Franck-Condon sidebands have been observed consistently is the longitudinal (stretching mode) vibration of a suspended single-wall carbon nanotube quantum dot $[9,13-$ 19]. Results range from the nanotube length dependence of the vibration frequency [9], or thermal occupation of a vibration mode [14], all the way to electronic pumping of nonequilibrium occupation [15], spin-vibron coupling [17], and a spin-dependent, electrostatically tunable electron-vibron coupling [19].

Here, we present observations of Franck-Condon sidebands at known absolute number $N_{\mathrm{el}}=1$ and $N_{\mathrm{el}}=2$ of conduction band electrons in the unperturbed carbon nanotube transport spectrum. The vibrational sidebands evolve only at finite axial magnetic field $B_{\|}$. The resulting millikelvin transport spectrum displays different sideband behavior depending on the electronic base state; the data indicates a valley-dependent Franck-Condon electron-vibron coupling parameter [4]. As a mechanism for the observed phenomenon, reshaping of the electronic wave function by the magnetic field [20] is discussed and modeled; the model manages to capture the essential observed behavior.

*Email address: andreas.huettel@ur.de

\section{CONDUCTION SIDE BANDS AT FINITE FIELD}

A sketch of the measured device is depicted in Fig. 1(a). Following Ref. [21], a carbon nanotube is grown over predefined rhenium contact electrodes and etched trenches. Subsequently, the device is cooled down in a top-loading dilution refrigerator and characterized electronically at a base temperature of $T_{\mathrm{mc}} \leqslant 30 \mathrm{mK}$, immersed into the diluted phase of the ${ }^{3} \mathrm{He} /{ }^{4} \mathrm{He}$ mixture. The length of the suspended nanotube segment is $L=700 \mathrm{~nm}$. The device has already been characterized electronically in Refs. [20,22,23]; as can also be seen in the current trace at low bias of Fig. 1(b), it displays the behavior of a small band-gap single-wall carbon nanotube, with transparent hole conduction and strong Coulomb blockade at low electron numbers. The first Coulomb oscillations exhibit very low current and require particular care to be resolved, see Fig. 1(c). Here, the opaque tunnel barriers are given by $p$ - $n$ junctions extended along the nanotube, between the electrostatically induced $n$-quantum dot and $p$-behavior near the leads [24,25].

In the following, we focus on the $1 \leqslant N_{\mathrm{el}} \leqslant 2$ transition, i.e., the second Coulomb oscillation at the electron side of the band gap. Figures 1(d) and 1(e) display the stability diagram close to the corresponding degeneracy point, for (d) $B_{\|}=0$ and for (e) a magnetic field $B_{\|}=10 \mathrm{~T}$ applied in parallel to the carbon nanotube axis. The overall conductance is very low; even so, the color scale in the figure has been cut off such as to focus on the substructure of the single electron tunneling (SET) regions. The strong black lines, corresponding to electronic excitations, shift with magnetic field; their detailed behavior, as well as the negative differential conductance at $B_{\|}=0$, is topic of ongoing analysis. The figures display a clear qualitative difference: While the areas between the electronic excitation lines are featureless in Fig. 1(d), in Fig. 1(e) they display a multitude of fine, closely spaced conductance resonances [see the arrows in Fig. 1(e)]. This also becomes visible in the comparison of the trace cuts of Fig. 1(f) $\left(B_{\|}=0 \mathrm{~T}\right)$ and Fig. $1(\mathrm{~g})\left(B_{\|}=10 \mathrm{~T}\right)$. 

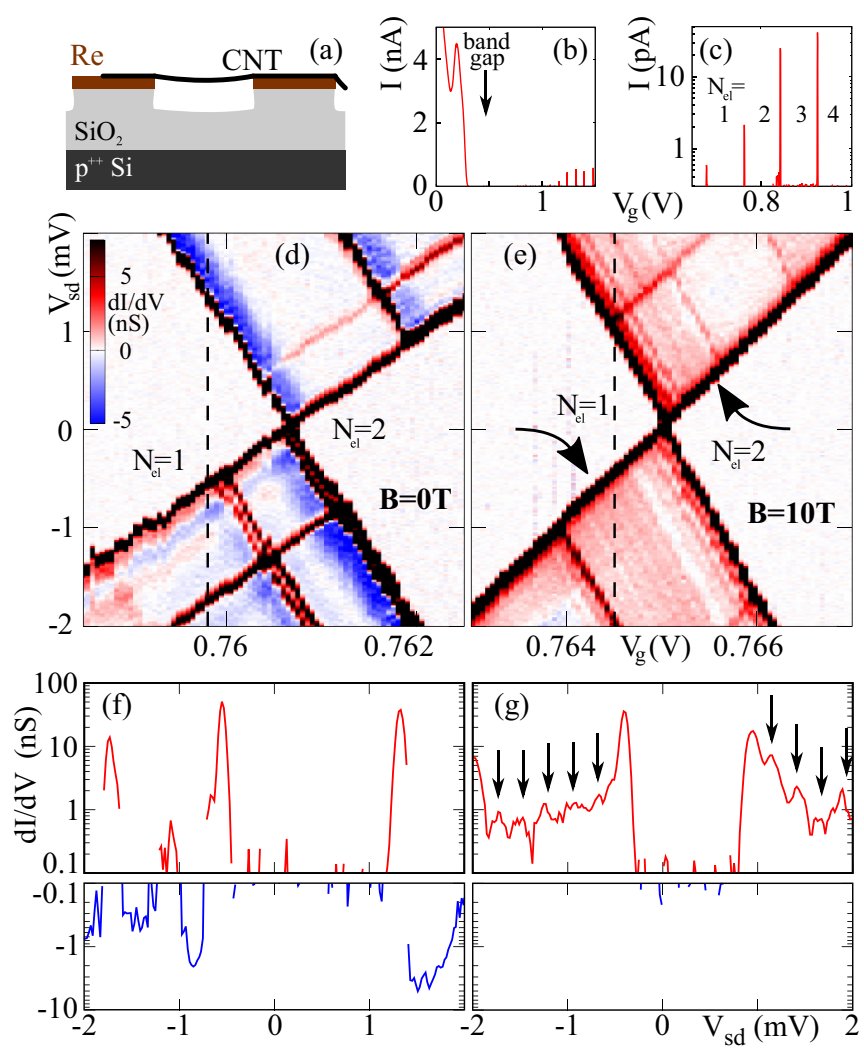

FIG. 1. (a) Schematic device geometry. A carbon nanotube is grown in situ across predefined rhenium contact electrodes and a trench. (b) Overview device characterization $I\left(V_{\mathrm{g}}\right)$ at $V_{\mathrm{sd}}=50 \mu \mathrm{V}$, showing transparent behavior in hole conduction, the band gap, and Coulomb oscillations in electron conduction. (c) Coulomb oscillations $I\left(V_{\mathrm{g}}\right)$ for $V_{\mathrm{sd}}=0.5 \mathrm{mV}$ near the band gap, with absolute electron numbers $N_{\mathrm{el}}$ marked. (d), (e) Differential conductance $\mathrm{d} I / \mathrm{d} V_{\mathrm{sd}}$ at the $1 \leqslant N_{\mathrm{el}} \leqslant 2$ transition, for a magnetic field of (d) $B_{\|}=$ 0 and (e) $B_{\|}=10 \mathrm{~T}$ parallel to the nanotube axis (identical color scale, cut off at $+7 \mathrm{nS}$ for better contrast). (f) Trace $\mathrm{d} I / \mathrm{d} V_{\text {sd }}\left(V_{\text {sd }}\right)$ at $B_{\|}=0 \mathrm{~T}, V_{\mathrm{g}}=0.7598 \mathrm{~V}$, see dashed line in (d), in logarithmic scale. The upper panel plots regions of positive $\mathrm{d} I / \mathrm{d} V_{\mathrm{sd}}$, the lower panel regions of negative $\mathrm{d} I / \mathrm{d} V_{\mathrm{sd}}$. (g) Trace $\mathrm{d} I / \mathrm{d} V_{\mathrm{sd}}\left(V_{\mathrm{sd}}\right)$ at $B_{\|}=10 \mathrm{~T}$, $V_{\mathrm{g}}=0.7645 \mathrm{~V}$, see dashed line in (e), using the same plotting method and scale as in (f). The equidistant arrows indicate harmonic excitation lines.

Figure 2(a) demonstrates the emergence of this phenomenon with increasing magnetic field. Here, we show the differential conductance $\mathrm{d} I / \mathrm{d} V_{\mathrm{sd}}\left(B_{\|}, V_{\mathrm{sd}}\right)$ as function of an applied field $B_{\|}$parallel to the carbon nanotube axis and of the bias voltage $V_{\text {sd }}$. The gate voltage is kept constant and chosen such that we trace across the $N_{\mathrm{el}}=1$ edge of the $1 \leqslant N_{\mathrm{el}} \leqslant 2$ SET region, see the inset of Fig. 2(a) for a sketch and Fig. 7(a) in Appendix A for a larger-scale plot of the conductance at the degeneracy point. Here and later, we focus our evaluation on the low magnetic field region since it provides a better signal/noise ratio.

For $B_{\|}=0$, the SET region edge, visible as line of differential conductance, is located at approximately $V_{\text {sd }}=0.8 \mathrm{mV}$. Due to a shift in energy of the electronic states involved in transport, it rapidly moves to higher bias voltages until $B_{\|} \simeq$ 1.5 $\mathrm{T}$ is reached. Here, the magnetic field induces a change
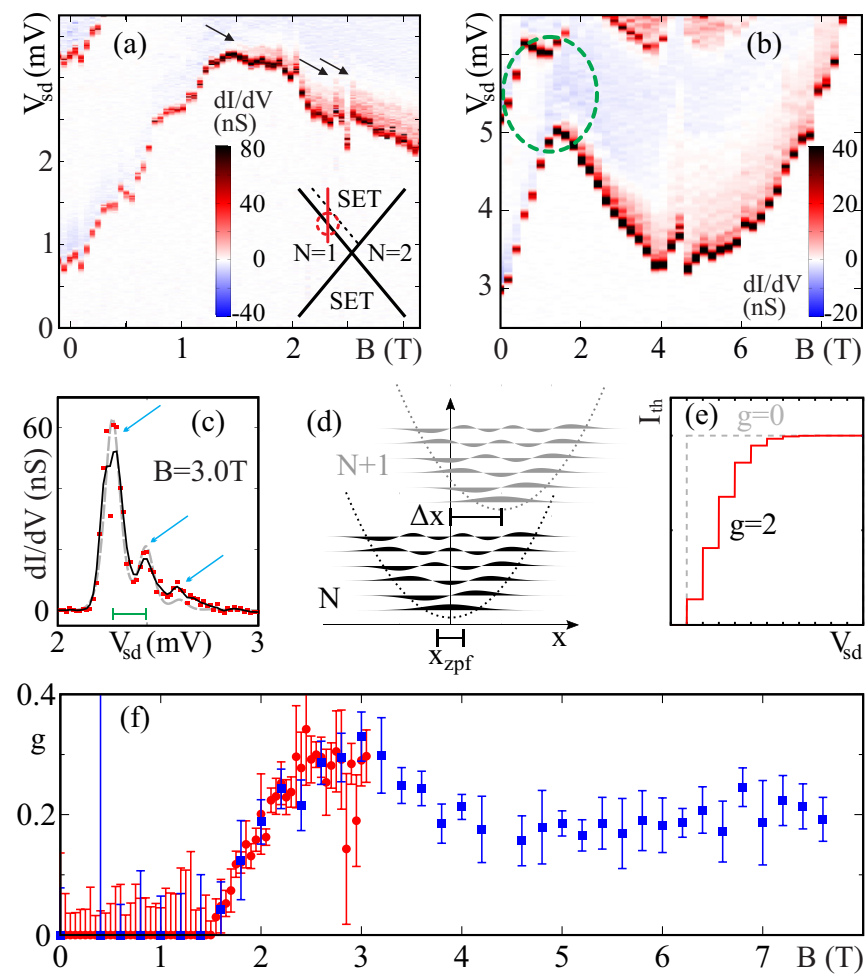

FIG. 2. (a) Differential conductance as function of magnetic field parallel to the nanotube axis $B_{\|}$and bias voltage $V_{\mathrm{sd}}, \mathrm{d} I / \mathrm{d} V_{\mathrm{sd}}\left(B_{\|}, V_{\mathrm{sd}}\right)$, at constant $V_{\mathrm{g}}=0.7599 \mathrm{~V}$. This cuts through the $1 \leqslant N_{\mathrm{el}} \leqslant 2$ single electron tunneling region, as sketched in the inset. (b) Similar measurement of $\mathrm{d} I / \mathrm{d} V_{\mathrm{sd}}\left(B_{\|}, V_{\mathrm{sd}}\right)$, covering a larger magnetic field range at lower resolution; $V_{\mathrm{g}}=0.758 \mathrm{~V}$. (c) Solid line: Smoothened trace $\mathrm{d} I / \mathrm{d} V_{\text {sd }}\left(V_{\text {sd }}\right)$ from (a) at $B_{\|}=3 \mathrm{~T}$, displaying the edge of the Coulomb blockade region, corresponding to the $1 \leqslant N_{\mathrm{el}} \leqslant 2$ ground state transition, and conductance side peaks; red points: unfiltered numerical derivative; gray dashed line: fit curve, see the text. (d) Illustration of the Franck-Condon coupling mechanism, see the text. (e) Schematic of the stepwise current increase at increasing bias voltage due to Franck-Condon coupling, here for $\Delta x=2 x_{\mathrm{zpf}}$ and thus $g=2$ as in (d). (f) Franck-Condon coupling parameter $g\left(B_{\|}\right)$ as function of the magnetic field; see Appendix B for details of the fit procedure. Red dots: data of (a); blue squares: data of (b).

in ground state, leading to a different energy dispersion. Soon afterward, sidebands of the differential conductance line emerge, see the arrows in Fig. 2(a). Figure 2(b) displays a larger parameter range than Fig. 2(a), though measured at reduced resolution. Still, the sidebands of the conductance line become clearly visible as an asymmetric broadening of the main conductance line (toward higher bias voltages).

An example trace cut from Fig. 2(a), both smoothened for clarity (black line) and as raw numerical derivative of the current (red points), is shown in Fig. 2(c). A manual analysis of the relative peak positions in each such recorded trace $I\left(V_{\text {sd }}\right)$ is given in Appendix A, see, in particular, Fig. 7(f). Its conclusion is that within the scatter the sidebands are equidistant within each trace. The excitation energy is magnetic-field independent for $B_{\|} \lesssim 6 \mathrm{~T}$ [26] and given by $\Delta \varepsilon \simeq 50 \mu \mathrm{eV}$. This indicates a harmonic oscillator independent of the electronic spectrum. Given its energy scale, we can identify it with the longitudinal vibration of the carbon nanotube [9]. 


\section{FRANCK-CONDON MODEL}

In multiple publications, the mechanism leading to vibrational harmonic sidebands in transport spectroscopy has been identified as the Franck-Condon principle [4,7,9]. As sketched in Fig. 2(d), the equilibrium position of the vibrational harmonic oscillator depends on the number of charges $N_{\mathrm{el}}$ on the nanotube; the rate of SET through its quantum dot is modified by the spatial overlap of the involved harmonic oscillator states $\left|\left\langle\Psi_{m}(N) \mid \Psi_{n}(N+1)\right\rangle\right|^{2}=\left|\left\langle\Psi_{m}(x) \mid \Psi_{n}(x+\Delta x)\right\rangle\right|^{2}$, with $m$ and $n$ as the vibrational quantum number at $N$ and $N+1$ electrons, respectively. $\Delta x$ is the displacement of the harmonic oscillator by the additional charge, cf. Fig. 2(d). The coupling strength is parametrized via the Franck-Condon coupling parameter $g=\left(\Delta x / x_{\mathrm{zpf}}\right)^{2} / 2$, comparing $\Delta x$ with the characteristic length scale of the harmonic oscillator $x_{\mathrm{zpf}}=$ $\sqrt{\hbar / m \omega}$.

As sketched in Fig. 2(e), a finite value of $g$ leads to a redistribution of current between vibrational state channels: At the bias voltage corresponding to the bare electronic transition energy, $N \longrightarrow N+1$ current is suppressed, but it increases whenever an additional vibration state becomes energetically available. A large number of extensions to this model has been developed to take into account specific details of transport spectra, see, e.g., Refs. [8,19,27-42]; however, for now we focus our analysis on the simplest theoretical case, assuming a single harmonic oscillator mode and fast relaxation into the vibrational ground state. In this case, the current step heights or conductance peak amplitudes follow the Poisson formula $[4,10], P_{n}=\left(e^{-g} g^{n}\right) / n !, \quad n=0,1,2, \ldots$, at an energy $\Delta E_{\mathrm{vib}}=n \hbar \omega$ from the bare electronic-state transition supplied via the bias voltage. The resulting step function of the current, in absence of broadening effects, is sketched in Fig. 2(e) for the example of a large $g=2$.

At base temperature, with $25 \mathrm{mK} k_{\mathrm{B}} \simeq 2 \mu \mathrm{eV}$, we expect the conductance lines to be lifetime-broadened, with a Lorentzian line shape. Thus, a sum of Lorentzians with amplitudes following the Poisson sequence and center points shifted by equidistant bias voltage offsets can be envisioned as model. In practice, it turns out that the Lorentzian line shape does not suit the measurement data well; this may indicate broadening effects beyond temperature and lifetime, as, e.g., fluctuating gate or bias voltages. Empirically, we choose the resonance shape of thermal broadening, $\alpha \cosh ^{-2}$, instead [43]. Details of the fit procedure can be found in Appendix B.

The result of evaluating the Franck-Condon coupling parameter $g$ for each trace $\mathrm{d} I / \mathrm{d} V_{\mathrm{sd}}\left(V_{\mathrm{sd}}\right)$ at fixed $B_{\|}$is plotted in Fig. 2(f). It shows the resulting magnetic-field dependence $g\left(B_{\|}\right)$for the $N_{\mathrm{el}}=1 \longrightarrow N_{\mathrm{el}}=2$ ground-state transition. The absence of sidebands for $B_{\|}<1.5 \mathrm{~T}$ corresponds to an absence of coupling, i.e., $g \sim 0$. For $B_{\|} \geqslant 1.5 \mathrm{~T}$, the coupling increases monotonously, reaching a maximum value $g\left(B_{\|}\right) \simeq$ 0.3 at $2.5 T \leqslant B_{\|} \leqslant 3 \mathrm{~T}$. Subsequently, we observe a slow decrease and stabilization at $g\left(B_{\|}\right) \simeq 0.2$.

\section{RELATION TO ELECTRONIC STATES}

To our best knowledge, no similar observations of a magnetic-field-dependent electron-vibron coupling have been published so far. Its onset at an anticrossing of electronic
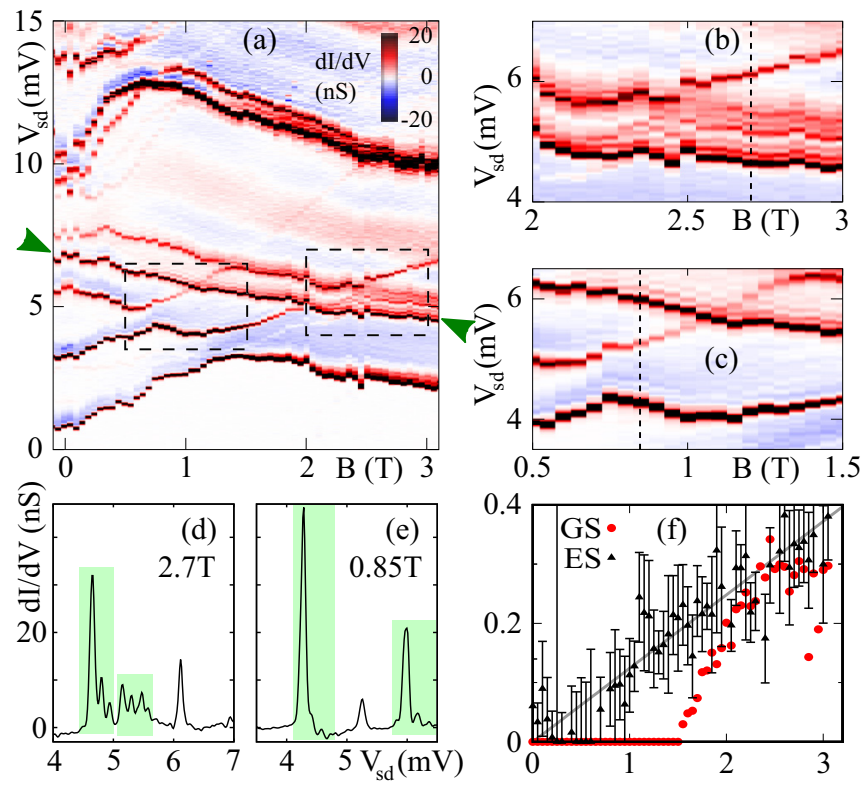

FIG. 3. (a) $1 \leqslant N_{\mathrm{el}} \leqslant 2$ excitation spectrum: Differential conductance $\mathrm{d} I / \mathrm{d} V_{\text {sd }}\left(B_{\|}, V_{\text {sd }}\right)$ as function of magnetic field $B_{\|}$and bias voltage $V_{\text {sd }}$, for constant gate voltage $V_{\mathrm{g}}=0.7599 \mathrm{~V}$. (b), (c) Detail enlargements (same color scale) of the areas marked with dashed rectangles in (a). (d), (e) Trace cuts $\mathrm{d} I / \mathrm{d} V_{\mathrm{sd}}\left(V_{\mathrm{sd}}\right)$ at the positions indicated in (b) and (c). Conductance peaks with clear vibrational sidebands are shaded. (f) Magnetic-field evolution of the FranckCondon coupling $g\left(B_{\|}\right)$for the excited-state transition marked with arrowheads in (a) (black), compared with the ground-state transition [red dots, same data as in Fig. 2(f)]. For the ground state, the error bars [already shown in Fig. 2(f)] have been omitted for clarity. The solid line is a linear fit after manual removal of outliers.

states, see the dashed ellipsoid in Fig. 2(b), suggests a connection to the electronic quantum numbers. As opposed to previous reports on the longitudinal vibration mode [9,13$15,17,19]$, here we are characterizing a device where the nanotube has grown cleanly across pre-existing contacts, and no (accidental or intentional) strongly inhomogeneuous potential distorts the wave functions in the suspended macromolecule away from the metallic contacts [20]. With this in mind, we have analyzed the vibrational sideband behavior of the electronic excited states in the transport spectrum.

A plot of the differential conductance at fixed gate voltage, as function of $B_{\|}$and the bias voltage $V_{\text {sd }}$, now over a large bias range, is shown in Fig. 3(a). A preliminary evaluation of the data of Fig. 3(a), taking also into account the one-electron excitation spectrum of the device [20], reveals two energetically close shells with both intra- and intershell exchange interaction [44]; detailed modeling of the two electron transport spectrum will be the topic of a separate work. Here, we limit ourselves to a straightforward classification of conductance lines by magnetic field dispersion; see Appendix $\mathrm{C}$ for the details.

The dominant magnetic field dependence of the electron energies in a carbon nanotube in an axial field originates from the electronic orbital magnetic moment $\mu_{\text {orb }}$, see, e.g., Refs. [20,45,46], and Appendix C. Thus, both one- and two-electron quantum states become at large field angular 

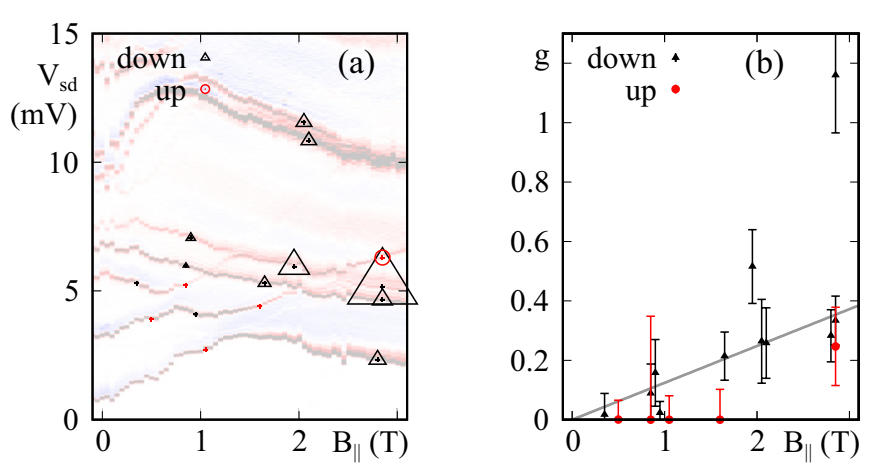

FIG. 4. Evaluation of the conductance resonances in Fig. 3(a) for harmonic sidebands; same data plotted in two different representations: (a) Coupling $g$ as symbol size (downward lines, black triangles; upward lines, red circles) for the parameter set $\left(B_{\|}, V_{\mathrm{sd}}\right)$ where it was evaluated, and superimposed on the resonance pattern (background); (b) coupling $g$ as function of magnetic field $B_{\|}$, irrespective of bias voltage. The solid line is the linear fit of Fig. 3(f).

momentum (and valley) eigenstates, and the slope of a conductance line in Fig. 3 indicates the angular momentum change when a second electron tunnels onto the quantum dot. If the state of the first electron remains unchanged, only the contribution of the second electron to the magnetic momentparallel or antiparallel to the magnetic field-determines the magnetic-field dispersion. From this, we can classify the conductance lines of Fig. 3(a). As expected, in the low-bias region of the figure, two dominant slopes clearly emerge; we identify these with the addition of a $\mathrm{K}^{\prime}$-valley electron (downward slope) or a K-valley electron (upward slope), respectively $[20,45,46]$.

Figures 3(b) and 3(c) enlarge the regions marked in Fig. 3(a) with dashed rectangles. Also here, the harmonic sidebands are immediately visible. However, at a first glance, only the downsloping spectral lines, where an electron is added in a $\mathrm{K}^{\prime}$-valley state, seem to exhibit electron-vibron coupling. Also the trace cuts at $B_{\|}=2.70 \mathrm{~T}$ [Fig. 3(d)] and $B_{\|}=0.85 \mathrm{~T}$ [Fig. 3(e)] demonstrate this, with the resonances accompanied by sidebands highlighted in green.

Extracting the Franck-Condon coupling parameter $g\left(B_{\|}\right)$ for an exemplary excited-state resonance [green arrowheads in Fig. 3(a)], using an identical fit procedure as for Fig. 2(f), we compare its evolution over the entire field range $0 \mathrm{~T} \lesssim B \lesssim$ $3 \mathrm{~T}$ with the two-electron ground state transition in Fig. 3(f). A finite $g$ persists to much lower magnetic field for the excited state transition; indeed, the plot shows a linear growth of $g\left(B_{\|}\right)$ in this range with $g\left(B_{\|}\right) \simeq 0.1241 / \mathrm{T} B_{\|}$(gray solid line). The sudden onset of coupling for the ground-state transition is thus consistent with a valley dependence and the change in ground state quantum numbers at $B_{\|} \simeq 1.5 \mathrm{~T}$, see the structure marked with a dashed ellipsoid in Fig. 2(b).

We have performed a systematic analysis of the conductance resonances in Fig. 3(a) by dividing them into segments at each crossing or anticrossing and evaluating each segment; details can be found in Appendix D. The result is shown in Fig. 4. Figure 4(a) displays symbols with their size representing the obtained $g$ parameter, superimposed on the line pattern of Fig. 3(a) at the parameters $\left(B_{\|}, V_{\mathrm{g}}\right)$ where the evaluation has

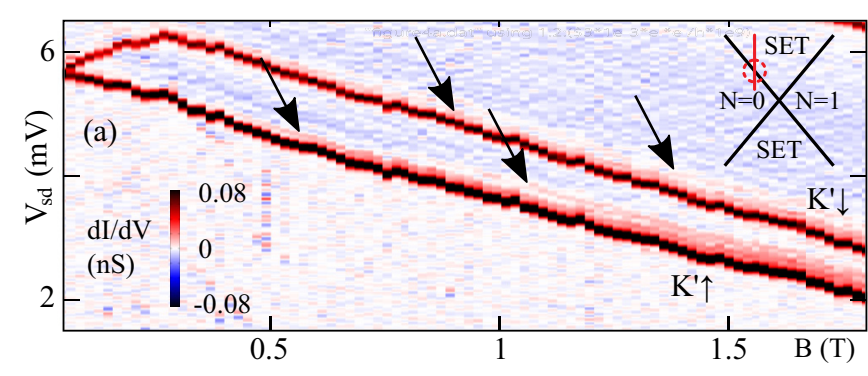

FIG. 5. Magnetic field behavior of the $0 \leqslant N_{\text {el }} \leqslant 1$ one electron ground-state transition. Traces of vibrational sidebands are indicated by arrows. Note that the color scale is cut off at $\pm 80 \mathrm{pS}$.

taken place. Figure 4(b) plots the value of $g$ as function of the selected magnetic field, irrespective of bias voltage $V_{\mathrm{sd}}$ and thus selected resonance line, with the fit function of Fig. 3(f) added. In both cases, red circles represent downsloping lines, black triangles upsloping lines. The result confirms our visual impression. In general, the upsloping lines, where an electron is added in a $\mathrm{K}$ state, display no vibrational sidebands, with the notable exception of the segment at $(3 \mathrm{~T}, 6 \mathrm{mV})$. The downsloping lines, where an electron is added in a $\mathrm{K}^{\prime}$ state, show a $g$ growing with magnetic field, in most cases following the same linear behavior as already observed in Fig. 3(f). One resonance line, also enlarged in Fig. 3(b), deviates with a large $g$.

It remains to clarify whether the observed effect is specific to the $1 \leqslant N_{\mathrm{el}} \leqslant 2$ region. Figure 5 displays the magneticfield dependence of a cut across the $0 \leqslant N_{\mathrm{el}} \leqslant 1$ ground-state transition. Also here, where an electron tunnels into a $\mathrm{K}^{\prime}$ state of the otherwise unoccupied conduction band, an onset of harmonic sidebands can be visually identified, for both spin alignments. Given the significantly lower conductance here, a more detailed evaluation turns out to be challenging. We can however conclude that the electron-vibron coupling is already inherent to single electron phenomena, and not limited to two-electron states [19]. This indicates a selectivity directly related to the single-particle valley quantum number.

\section{DISCUSSION}

The transport spectra were measured with the carbon nanotube immersed into the ${ }^{3} \mathrm{He} /{ }^{4} \mathrm{He}$ mixture (D phase) of the dilution refrigerator. Its viscosity at base temperature, $\eta \sim$ $10^{-5} \mathrm{~N} \mathrm{~s} / \mathrm{m}^{2}$ [47], is sufficiently high to mechanically dampen the transversal vibration mode [48]. As observed here, this does not affect the longitudinal mode; a likely explanation is that motion along the nanotube axis does not require any displacement of liquid.

In literature, Weber et al. [19] have reported an electronic state dependent Franck-Condon coupling in a $N_{\mathrm{el}}=4 n+2$ quantum dot, switchable via a local gate potential deformation. They apply a magnetic field perpendicular to the nanotube, and see no effect of that field on the vibrational sidebands. Due to valley mixing and the field direction, their data is in the regime of bonding and antibonding valley linear combinations. A different electron-vibron coupling of (valleyground state) spin singlet and (valley-distributed) spin triplet 
states, as observed there, obviously can also indicate a valleydependent effect. Whether this is related to our results still requires further analysis.

Models discussing strong electron-vibron coupling in carbon nanotube quantum dots typically assume an inhomogeneuous charge distribution relative to the vibration mode envelope, or more generally, a different localization of electron and vibron wave function, see, e.g., Refs. [9,30,32,37]. This is consistent with the occurrence of Franck-Condon sidebands in devices with local gates close to the nanotube $[14,18,19]$. In previous devices without such an electrode, a local potential may have been introduced via fabrication defects or impurities on the nanotube $[9,13,15]$. These explanations do not lend themselves for the device presented here, displaying a highly regular one electron spectrum [20] and a regular addition spectrum over a large electron number range $[22,49]$. Nevertheless, the formation of the quantum dot via gate-induced $p$ - $n$ junctions, see, e.g., Refs. [24,25], leads to an electronic confinement more narrow than the mechanically active device length, and typically centered within the suspended nanotube segment.

Comparison with existing theoretical $[30,37]$ and experimental works [19] then suggests that the axial magnetic field modifies the Franck-Condon coupling $g$ by shifting the electronic wave function relative to the vibron envelope. A valley-dependent mechanism having this effect was recently proposed in Ref. [20], based on Refs. [50-52]. Essentially, the axial magnetic field introduces an Aharonov-Bohm phase around the nanotube. Due to the bipartite graphene lattice, the axial component $\kappa_{\|}$and the circumferential component $\kappa_{\perp}$ of an electron-state wave vector are coupled; one example solution is plotted in Fig. 6(a) following Ref. [20]. The axial magnetic field thus also modifies the longitudinal boundary conditions and thereby the charge distribution along the carbon nanotube axis: the wave-function envelope for each graphene sublattice is tuned from a $\lambda / 4$ shape, with a finite value at one edge, near $B_{\|}=0$ to a $\lambda / 2$ shape, as expected for a traditional "quantum box", at large field; see the drawings in Fig. 6(a).

\section{VARIABLE COUPLING MODEL}

Based on the arguments brought forward in the previous section, we have constructed a toy model following Refs. [20,37]. Details can be found in Appendix E; our steps are summarized as follows: we simplify the electronic wavefunction amplitudes on the two graphene sublattices to be of the shape $\psi \propto \sin (\pi \phi x)$ over the length of the quantum dot, where $\phi \in[1,2]$ is used to continuously tune the wave function shape from one antinode to two antinodes and thus approximate the impact of the magnetic field. The total linear charge density $\rho(x)$ is obtained by summing up the charge densities $\propto|\psi|^{2}$ of the sublattices, one being the spatial mirror image of the other [20]. The vibron is equally simplified as having a deflection amplitude $u(x) \propto \sin (\pi x)$.

Given the strong confinement of our electronic states and the low characteristic energy of our harmonic excitations, the quantum dot occupies likely a smaller part of the nanotube than the vibron. Figure 6(b) shows both charge density and vibron envelope for a length ration $L_{d} / L_{v}=1 / 2$ and for a
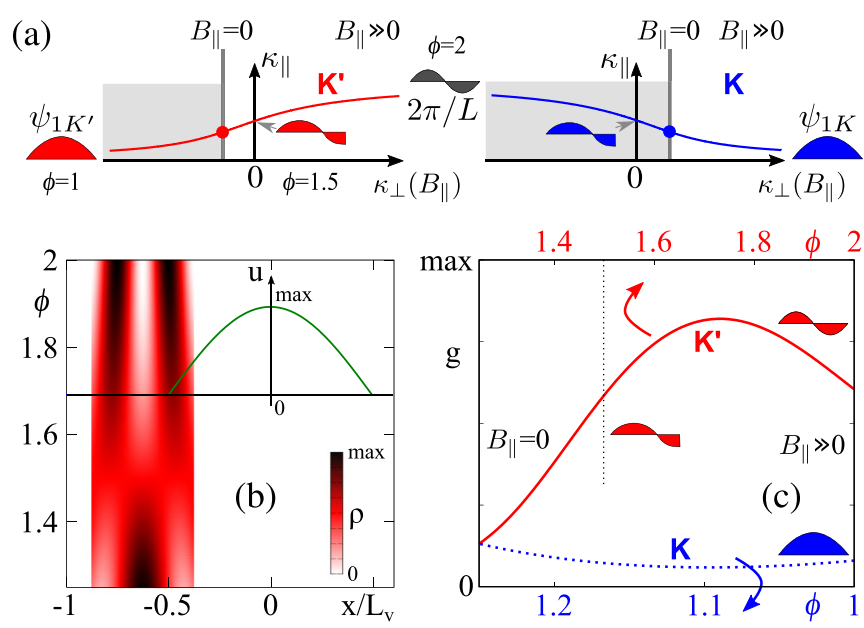

FIG. 6. Variable coupling model construction. (a) Low-energy wave function solutions for electrons on one of the carbon nanotube sublattices, in the case of hard longitudinal confinement and cross quantization, following Ref. [20]. Solid lines plot the allowed wave vector $\left(\kappa_{\perp}, \kappa_{\|}\right)$, schemata the resulting envelope of the sublattice wave function for a $\mathrm{K}^{\prime}$ and a $\mathrm{K}$ state. An axial magnetic field modifies the boundary conditions, tuning $\kappa_{\perp}$ toward higher values. (b) Example arrangement of a vibron and a quantum dot. Green line: Vibron amplitude envelope, length $L_{v}=1$. Color plot, charge density of a quantum dot of length $L_{d}=L_{v} / 2$, summed over both sublattices, as function of position and a parameter $\phi$ that tunes the per-sublattice wave function shape from one antinode $(\phi=1)$ to two antinodes $(\phi=2)$. (c) Approximation for the Franck-Condon parameter $g$ as function of $\phi$ and thereby the magnetic field $B_{\|}$, see the text.

shift in center position $x_{d}-x_{v}=-0.625 L_{v}$. The charge density is plotted as function of both the position and $\phi$, for the range $\phi \in[1.25,2]$ approximately covered by a $\mathrm{K}^{\prime}$ state when the axial magnetic field is tuned from zero to large values, cf. Fig. 6(a) and Ref. [20].

Following Ref. [37], we assume the interaction energy between linear charge density and deformation potential to be

$$
E_{\mathrm{ev}} \propto \int \rho(x) \frac{\mathrm{d} u}{\mathrm{~d} x} \mathrm{~d} x
$$

and the Franck-Condon parameter to be $g \propto\left|E_{\text {ev }}\right|^{2}$. The result for $g(\phi)$ is plotted in Fig. 6(c), see the red solid line. Recalling that $\phi=2$ here describes the asymptotic limit of large $B_{\|}$, the result qualitatively agrees with the behavior of $g$ for a $\mathrm{K}^{\prime}$ state in our experiment, cf. Fig. 3(f) for the low-field and Fig. 2(f) for the high-field behavior. For the same magnetic field range, a $\mathrm{K}$ state covers the range $\phi \in[1,1.25]$, with the large field limit $\phi=1$. Here, the coupling further decreases and remains small, see the dotted blue line.

Despite the many simplifications, this model describes the essential observations of the measurement. For a $\mathrm{K}^{\prime}$ state, the Franck-Condon coupling increases from near zero with magnetic field, reaches a maximum, and then for large field becomes constant at smaller value. For a $\mathrm{K}$ state, the coupling remains low. The sudden onset of coupling for the ground state around $B_{\|}=2 \mathrm{~T}$, Fig. 2(f), is due to a transition between these two cases. Limitations of the model become clear, however, when we look at the size ratio and placement of electron 
and vibron. In an ultraclean, suspended nanotube device, we expect the electronic system to be approximately centered in the suspended part. The vibron should cover at least the entire suspended part, which speaks against a spatial arrangement as shown in Fig. 6(b). A parameter search using our toy model has not yielded fundamentally different situations with suitable $g\left(B_{\|}\right)$behavior so far. This shall likely require a more realistic and in particular also quantum mechanical treatment.

\section{CONCLUSIONS}

In conclusion, we demonstrate that vibrational sidebands emerge in the $0 \leqslant N_{\mathrm{el}} \leqslant 2$ transport spectrum of an ultraclean nanotube at a finite magnetic field parallel to the nanotube axis. The sidebands are equidistant, with an oscillator quantum field-independent for $B_{\|} \lesssim 6 \mathrm{~T}$. Their evaluation results in a field-dependent Franck-Condon coupling parameter $g\left(B_{\|}\right)$. Our data indicate that, predominantly, conductance lines corresponding to the addition of a $\mathrm{K}^{\prime}$ valley electron develop a finite coupling parameter $g$. A tentative mechanism for this can be a field-induced and valley-selective modification of the electronic wave-function envelope [20]. Following Ref. [37], we have developed a simplified classical model and are able to reproduce the essential behavior of $g$. A realistic match of device and model parameters will require a more detailed theoretical treatment.

It has long been proposed that, similar to existing experiments using the transversal vibration [53], the longitudinal vibration coupling in a carbon nanotube Franck-Condon system can also be controlled via changing the charge distribution along the nanotube [30,37]. With our experimental results supporting this idea, the integration of the longitudinal vibration mode into quantum technological applications and its targeted manipulation as quantum harmonic oscillator [15] now becomes an interesting challenge.

\section{ACKNOWLEDGMENTS}

We would like to thank M. Margańska, M. Grifoni, A. Donarini, Ch. Strunk, E. A. Laird, and P. Hakonen for insightful discussions, and Ch. Strunk and D. Weiss for the use of experimental facilities. The data has been recorded using the LAB::MEASUREMENT software package [54]. The authors acknowledge financial support by the Deutsche Forschungsgemeinschaft (Emmy Noether Grant Hu 1808/1, GRK 1570, SFB 689, and SFB 1277) and by the Studienstiftung des deutschen Volkes.

The device was fabricated by P.L.S. and D.R.S; the lowtemperature measurements were performed by all authors jointly. The resulting data was evaluated by P.L.S. and A.K.H.; the paper was written and the project was supervised by A.K.H.

\section{APPENDIX A: HARMONICITY AND EXCITATION ENERGY}

Figures 7(a) and 7(b) display the conductance near the $1 \leqslant N_{\mathrm{el}} \leqslant 2$ degeneracy point, for $B_{\|}=0 \mathrm{~T}$ and $B_{\|}=5 \mathrm{~T}$ and over a large bias voltage range $\left|V_{\text {sd }}\right| \leqslant 10 \mathrm{mV}$. The magnetic field dependent measurements evaluated in Figs. 2 and 3 correspond to traces taken across the SET region, in the parameter range indicated in Fig. 7(a) by a yellow marker. Part of such a trace $\mathrm{d} I / \mathrm{d} V_{\mathrm{sd}}\left(V_{\mathrm{sd}}\right)$ for constant $B_{\|}$and $V_{\mathrm{g}}$ is shown schematically in Fig. 7(c). From it, the peak distances $\Delta V_{\mathrm{sd} 1}$, $\Delta V_{\mathrm{sd} 2}, \Delta V_{\mathrm{sd} 3}$ as indicated in the drawing can be extracted. They correspond to excitation energies $\Delta \varepsilon_{1}, \Delta \varepsilon_{2}, \Delta \varepsilon_{3}$.

In the stability diagrams, conductance lines ending at the $N_{\mathrm{el}}=2$ Coulomb blockade region (i.e., for $V_{\mathrm{sd}}>0$ with negative slope) correspond to two-electron excitations; lines ending at the $N_{\mathrm{el}}=1$ Coulomb blockade region (i.e., for $V_{\text {sd }}>0$ with negative slope) correspond to one-electron excitations. In the evaluated parameter region, only lines with negative slope are visible. For them, the conversion from bias voltage distances $\Delta V_{\text {sd }}$ to excitation energies $\Delta \varepsilon$, based on the capacitances in the quantum dot system, can be illustrated by the sketch of Fig. 7(b). Given the slopes $\Delta V_{\mathrm{sd}} / \Delta V_{\mathrm{g}}$ of the two edges of the SET region in the stability diagram, $s_{1}<0$ and $s_{2}>0$, as indicated in the sketch, the conversion factor $f\left(B_{\|}, V_{\mathrm{g}}\right)$ can be derived from elementary geometry as

$$
\Delta \varepsilon=\frac{1}{1-\left(s_{1} / s_{2}\right)} e \Delta V_{\mathrm{sd}}=f\left(B_{\|}, V_{\mathrm{g}}\right) e \Delta V_{\mathrm{sd}}
$$

Here we indicate with $f\left(B_{\|}, V_{\mathrm{g}}\right)$ that the factor can change with magnetic field and gate voltage due to modification of the electronic wave function shapes and thus the charge distributions and capacitances. The value of $f$, as extracted from stability diagrams at $B_{\|}=0,5,10 \mathrm{~T}$, is plotted in Fig. 7(c), with a linear fit added. In the plotted range, $f$ varies by only approximately $10 \%$.

The result of a manual evaluation of the data of Fig. 2(b), where peak positions have been read out from line plots, is shown in Fig. 7(d). Here, the red squares correspond to the peak distance of the first sideband relative to the base conductance resonance, the black dots to the one of the second sideband relative to the first sideband, and the blue triangles to the one of the third sideband relative to the second sideband. The left axis displays distance in bias voltage, the right axis the value converted into energy, using constant $f(5 \mathrm{~T})$ for simplicity. Within the scatter, the three types of points lie in the same band of values, indicating equidistant quantum states and thus harmonic oscillator behavior. The oscillator quantum is for $0 \mathrm{~T} \leqslant B_{\|} \leqslant 6 \mathrm{~T}$ approximately constant at $\Delta V_{\mathrm{sd}} \simeq 0.15 \mathrm{mV}$, corresponding to $\Delta \varepsilon=\hbar \omega \simeq 50 \mu \mathrm{eV}$; this is the region predominantly discussed in the main text.

For larger $B_{\|}$, the data points seem to indicate a gradual increase in $\Delta \varepsilon$. Given the decreasing signal-to-noise ratio and limited data for this field range, it is unclear whether this is a real effect. Surprisingly, the stability diagram at $B_{\|}=10 \mathrm{~T}$, Fig. 1(e), displays $\Delta V_{\text {sd }} \simeq 0.25 \mathrm{mV}$, which would confirm such an increase.

The theoretical value for the energy quantum of the carbon nanotube longitudinal vibration is given by [9]

$$
\Delta \varepsilon_{\mathrm{th}}=\frac{h}{L} \sqrt{\frac{Y}{\rho}},
$$

where $L$ is the nanotube length, $Y$ is Young's modulus, and $\rho$ is the nanotube mass density. Assuming $\rho=1.3 \mathrm{~g} / \mathrm{cm}^{3}$ and 

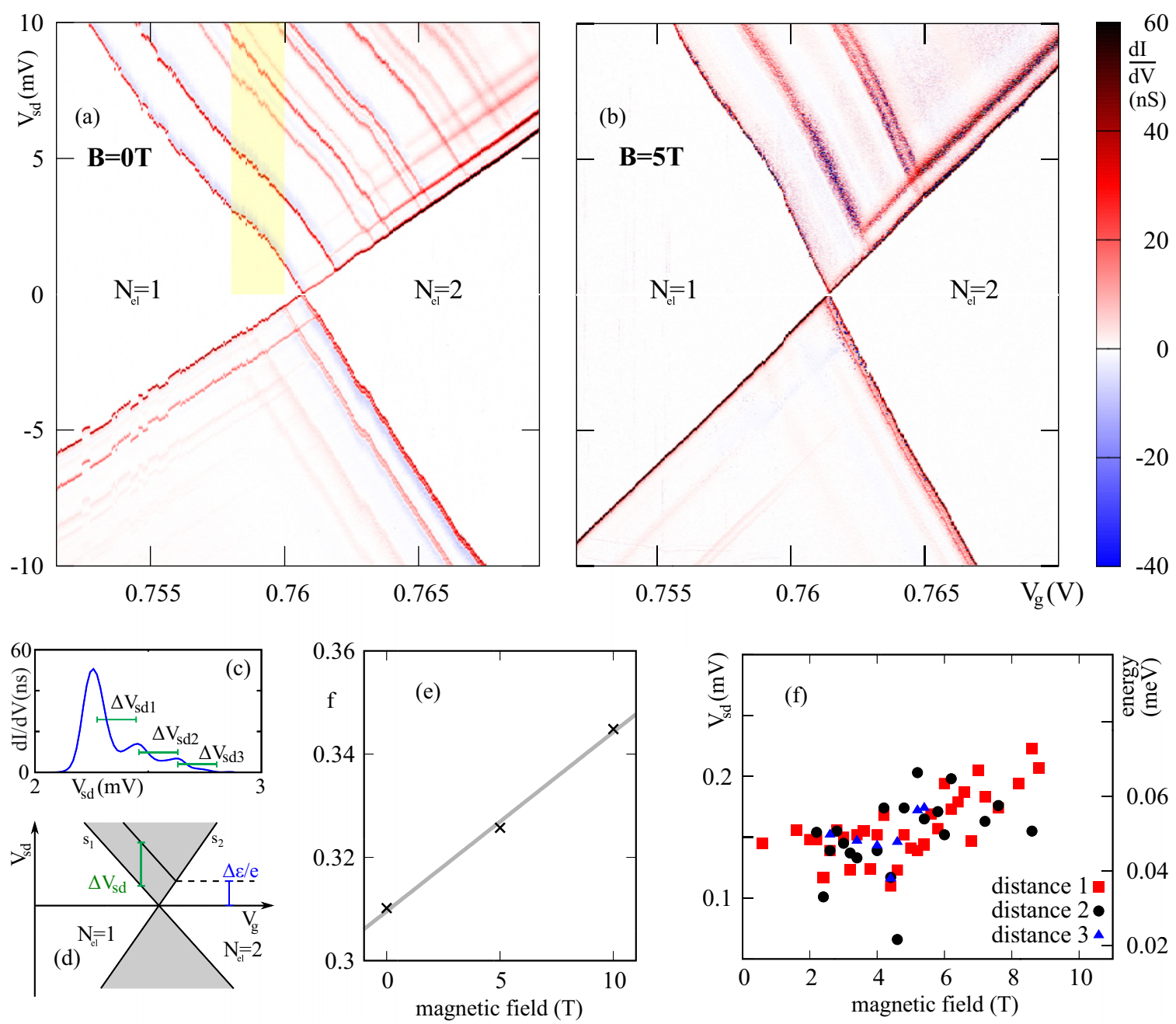

FIG. 7. (a) Large bias voltage range stability diagram $\mathrm{d} I / \mathrm{d} V_{\mathrm{sd}}\left(V_{\mathrm{g}}, V_{\mathrm{sd}}\right)$ of the $1 \leqslant N_{\mathrm{el}} \leqslant 2$ transition at $B_{\|}=0 \mathrm{~T}$. Figure 1 (d) is a detail zoom of this plot. The parameter region of the trace cuts evaluated in Figs. 2 and 3 is shaded in yellow. (b) Large bias voltage range stability diagram $\mathrm{d} I / \mathrm{d} V_{\text {sd }}\left(V_{\mathrm{g}}, V_{\text {sd }}\right)$ at $B_{\|}=5 \mathrm{~T}$. (c) Illustrative example trace $\mathrm{d} I / \mathrm{d} V_{\text {sd }}\left(V_{\text {sd }}\right)$ for constant $B_{\|}$and $V_{\mathrm{g}}$. (d) Using the slopes $\Delta V_{\text {sd }} / \Delta V_{\mathrm{g}}$ of the two edges of the single electron tunneling region in the stability diagram, $s_{1}<0$ and $s_{2}>0$, the bias differences $\Delta V_{\mathrm{sd}}$ can be converted to energy differences $\Delta \varepsilon$ (here demonstrated for a $N_{\mathrm{el}}=2$ state). (e) Conversion factor $f\left(B_{\|}\right)$, defined via $\Delta \varepsilon=f\left(B_{\|}\right) \cdot e \Delta V_{\text {sd }}$, as extracted from the stability diagrams at $B_{\|}=0 \mathrm{~T}$ [see (a), Fig. 1(d)], $B_{\|}=5 \mathrm{~T}$ [see (b)], and $B_{\|}=10 \mathrm{~T}$ [see Fig. 1(e)]. (f) Manually extracted distances between conductance peak positions, cf. (a), using the data set of Fig. 2(b). Left axis: Raw distances in bias voltage, right axis: distances converted to energy, using $f(5 \mathrm{~T})$.

$Y=1 \mathrm{TPa}$, this results in [9]

$$
\Delta \varepsilon_{\mathrm{th}} \approx \frac{0.11 \mathrm{meV}}{L(\mu \mathrm{m})} .
$$

For our device, using the contact separation of $L=0.7 \mu \mathrm{m}$ as approximate value of the suspended nanotube segment length, we obtain $\Delta \varepsilon_{\text {th }} \simeq 160 \mu \mathrm{eV}$. While there is a clear deviation, our measurement still lies at the edge of the typical scatter of oscillator quanta observed in experimental literature, see Fig. 8 for an overview.

\section{APPENDIX B: FRANCK-CONDON MODEL AND FIT PROCEDURE}

In the context of SET through a carbon nanotube, the Franck-Condon coupling parameter $g$ describes the spatial shift of the nanotube equilibrium position as harmonic oscillator when an additional electron is added to it. It is given by $g=\left(\Delta x_{0} / x_{\mathrm{zpf}}\right)^{2} / 2$, where $\Delta x_{0}$ is the shift in equilibrium position, $\Delta x_{0}=x_{0}(N+1)-x_{0}(N)$. In the denominator, $x_{\mathrm{zpf}}=$ $\sqrt{\hbar /(m \omega)}$ is the characteristic length scale of the harmonic oscillator, describing the wave function extension of the ground state and/or its zero point fluctuations. For $\hbar \omega=50 \mu \mathrm{eV}$ and $m=1.3 \times 10^{-21} \mathrm{~kg}$ [55], we obtain $x_{\mathrm{zpf}}=1.0 \mathrm{pm}$.

At finite temperature, a harmonic oscillator can both absorb and emit vibrons. Here, in the limit of low temperature and fast vibrational relaxation compared to the tunnel rates, we assume that for any SET process we start out in the $N_{\mathrm{el}}$ electron vibrational ground state. For each number of vibration quanta $n$, there is a distinct overlap $\left|\left\langle\Psi_{0}\left(N_{\mathrm{el}}\right) \mid \Psi_{n}\left(N_{\mathrm{el}}+1\right)\right\rangle\right|^{2}$ of the $N_{\mathrm{el}}$ electron vibrational ground state with an $N_{\mathrm{el}}+1$ electron, $n$ vibron state. This leads to a series of equidistant steps in current $I\left(V_{\mathrm{sd}}\right)$ or peaks in differential conductance 

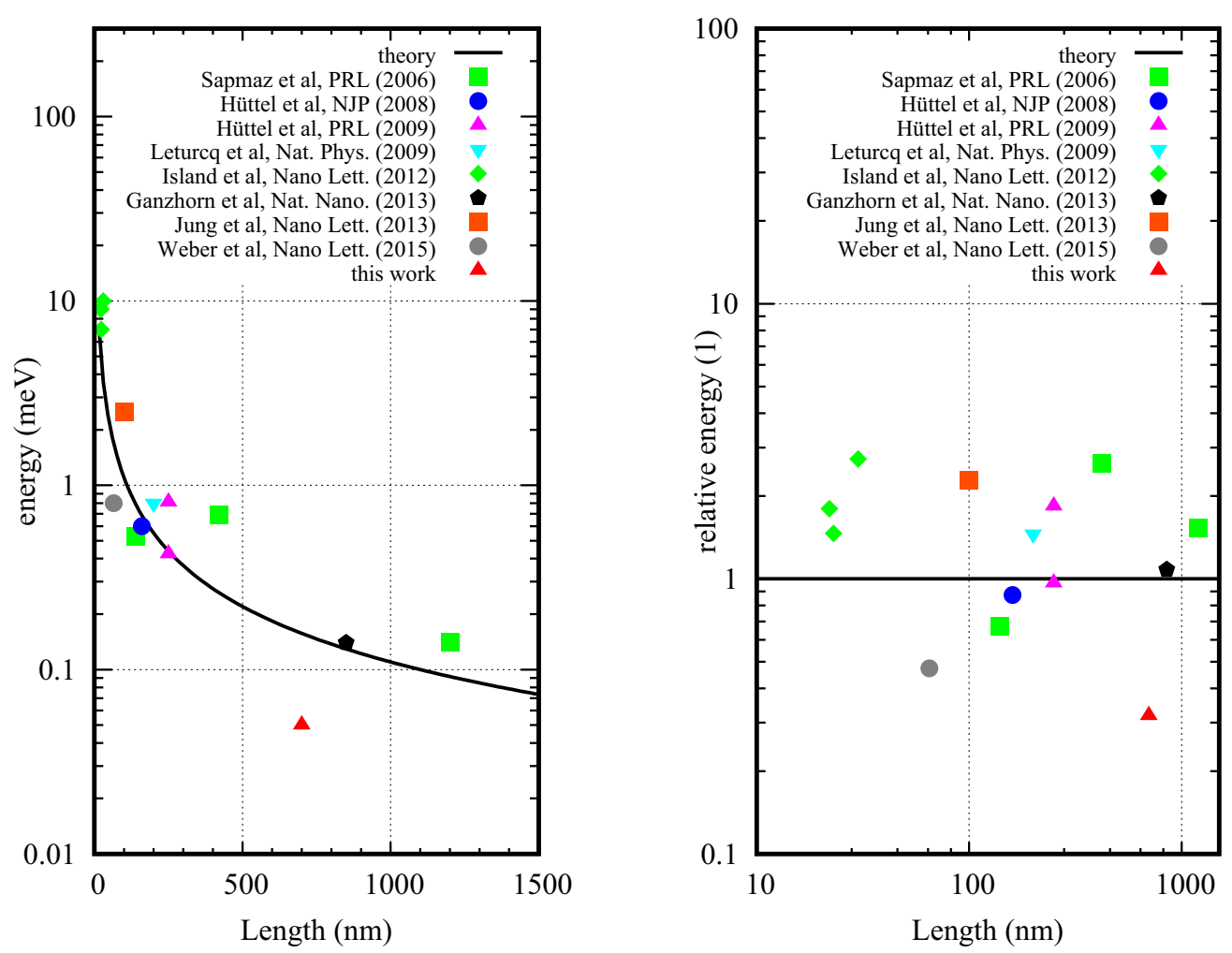

FIG. 8. Overview of carbon nanotube longitudinal vibration oscillator quanta $\Delta \varepsilon=\hbar \omega$ observed in published literature [9,13-19] as function of device length, in comparison with the theoretical result $\Delta \varepsilon_{\mathrm{th}}(L)=110 \mathrm{meV} / L[\mathrm{~nm}]$. Left: Unscaled $\Delta \varepsilon(L)$, linear length scale; right: $\Delta \varepsilon(L) / \Delta \varepsilon_{\mathrm{th}}(L)$, logarithmic length scale.

$\mathrm{d} I / \mathrm{d} V_{\mathrm{sd}}\left(V_{\mathrm{sd}}\right)$, whenever sufficient energy for reaching the next vibrational state becomes available.

The contributions of the vibrational states and thus the current step heights are given for the limit of low temperature and fast vibrational relaxation by the Poisson formula $[4,10]$,

$$
\Delta I_{n} \propto \frac{e^{-g} g^{n}}{n !}, \quad n=0,1,2, \ldots
$$

at an energy $\varepsilon=n \hbar \omega$ from the bare electronic state transition. The resulting step function of the current, in the idealized case of total absence of effects such as thermal or lifetime broadening, is shown in Figs. 9(a)-9(c) for different values of $g$.

In a measurement, the steps will be broadened due to finite temperature, finite lifetime of the involved quantum states, and additional mechanisms such as, e.g., potential fluctuations. As an approximation, we assume that this broadening equally affects all steps. Then, the differential conductance $G=\mathrm{d} I / \mathrm{d} V_{\text {sd }}$ exhibits a sequence of peaks, each corresponding to one step in current, with conductance peak heights proportional to the current step heights. Even though $G$ is in our measurement a derived quantity, it is both a better base for visualization of the phenomena, see the figures of this paper, and a better base for numerical curve fitting than the measured current. To minimize the impact of postprocessing, we use for the fits the raw numerically differentiated conductance $\Delta I / \Delta V_{\text {sd }}$, without any smoothing or other numerical filtering applied.

As already mentioned in the main text, we find that our data is fitted well using the typical shape of a thermally broadened
Coulomb oscillation,

$$
G_{\mathrm{th}}\left(V_{\mathrm{sd}}, V_{\mathrm{sd}}^{0}\right)=\left[\cosh \left(\frac{V_{\mathrm{sd}}-V_{\mathrm{sd}}^{0}}{\gamma}\right)\right]^{-2},
$$

where $\gamma$ describes the peak width. For our fit model, we sum up a sequence of these peaks, weighted according to Eq. (B1),
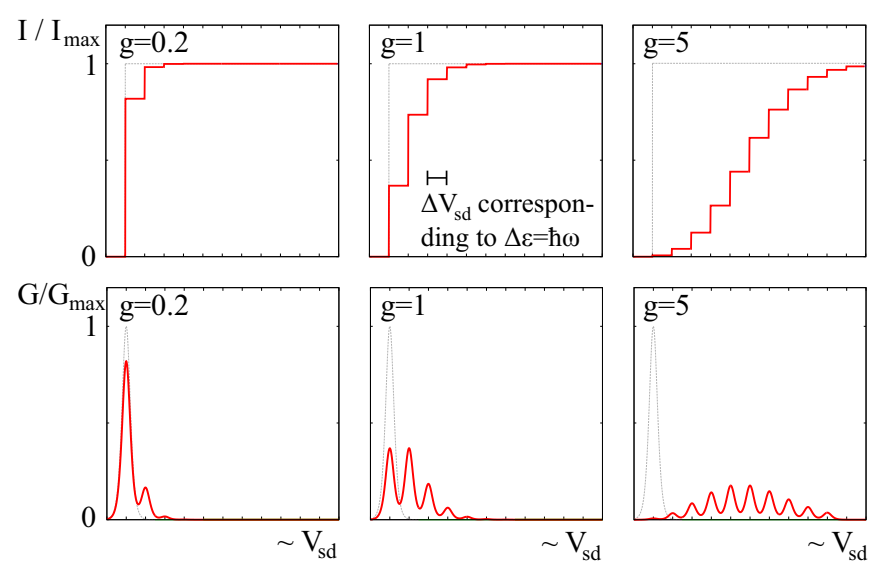

FIG. 9. Sketch of the impact of the Franck-Condon coupling parameter $g$ on single electron tunneling: (a)-(c) current $I\left(V_{\mathrm{sd}}\right)$, in an idealized system where no broadening (thermal, lifetime, or otherwise) is present, for (a) $g=0.2$, (b) $g=1$, and (c) $g=5$; (d)-(f) conductance at finite broadening, see the text. In each panel, the case of no electron-vibron interaction $(g=0)$ is overlaid as gray dashed line. 
cutting off at $n=10$ since subsequent terms are negligible for $g \lesssim 1$, and normalize such that the maximum conductance $G_{\text {max }}$ at the electronic base resonance $(n=0)$ can be used as convenient fitting parameter. This results in

$$
G\left(V_{\mathrm{sd}}, V_{\mathrm{sd}}^{0}\right)=G_{\max } \frac{\sum_{n=0}^{9} P_{n} G_{\mathrm{th}}\left(V_{\mathrm{sd}}, V_{\mathrm{sd}}^{0}+n \Delta V_{\mathrm{sd}}\right)}{\sum_{n=0}^{9} P_{n} G_{\mathrm{th}}\left(V_{\mathrm{sd}}^{0}, V_{\mathrm{sd}}^{0}+n \Delta V_{\mathrm{sd}}\right)} .
$$

Given the combination of large scatter in the data, see, e.g., Fig. 2(c), and the large number of free fit parameters $\left[V_{\mathrm{sd}}^{0}, G_{\mathrm{max}}, \gamma, g, \Delta V_{\mathrm{sd}}=\hbar \omega /(e f)\right]$ the fit displays instabilities and large error bars. However, it turns out that the fit results for both $\gamma$ and $\Delta V_{\text {sd }}$ scatter in the evaluated regions around field-independent values [also validating our manual evaluation of Fig. 7(d)]. As a consequence, we fix both parameters to an average value and rerun the fit with a reduced set of free parameters $\left(V_{\mathrm{sd}}^{0}, G_{\max }, g\right)$. To give an insight into the results of this procedure, Fig. 10 shows for part of the evaluation both the raw data and the best fit functions; these correspond to data traces of Fig. 2(b) and the fit prarameters plotted in Fig. 2(f) as blue squares.

\section{APPENDIX C: CLASSIFICATION OF EXCITED STATE RESONANCES}

In a magnetic field at an angle $\varphi$ to the carbon nanotube axis, the linearized single particle Hamiltonian of an electron in a carbon nanotube quantum dot close to the Dirac point is given by $[20,49]$

$$
\begin{aligned}
\hat{H}_{\mathrm{CNT}}= & \underbrace{\varepsilon_{d} \hat{I}_{\sigma} \otimes \hat{I}_{\tau}}_{\text {"shell" }}+\underbrace{\frac{\Delta_{\mathrm{KK}}}{2} \hat{I}_{\sigma} \otimes \hat{\tau}_{x}}_{\text {valley mixing }}+\underbrace{\frac{\Delta \mathrm{SO}}{2} \hat{\sigma}_{z} \otimes \hat{\tau}_{z}}_{\text {spin-orbit interaction }} \\
& +\underbrace{\frac{g_{\mathrm{s}} \mu_{\mathrm{B}}|\vec{B}|}{2}\left(\cos \varphi \hat{\sigma}_{z}+\sin \varphi \hat{\sigma}_{x}\right) \otimes \hat{I}_{\tau}}_{\text {Zeeman effect }} \\
& +\underbrace{g_{\text {orb } \mu_{\mathrm{B}}|\vec{B}| \cos \varphi \hat{I}_{\sigma} \otimes \hat{\tau}_{z}}}_{\text {orbital angular moment }}
\end{aligned}
$$

Here, the Pauli matrices $\hat{\sigma}_{i}$ act on the spin part of the wave function, and the Pauli matrices $\hat{\tau}_{j}$ on the orbital/valley part of the wave function, respectively; the basis is given by spin and valley eigenstates in axial direction.

For a field alignment parallel to the nanotube $(\varphi=0)$, already at moderate field (e.g., $B_{\|} \sim 0.5 \mathrm{~T}$ [20]) the contribution of the orbital angular moment exceeds the valley mixing and the eigenstates of the Hamiltonian become spin and valley eigenstates, with spin and orbital angular moment aligned parallel to the nanotube axis. With $\sigma= \pm 1$ and $\tau=$ \pm 1 describing spin and orbital angular moment directions, the magnetic-field-dependent terms of Eq. (C1) then lead to the energy contribution:

$$
E_{\sigma \tau}\left(B_{\|}\right)=\left(\sigma \frac{g_{\mathrm{s}} \mu_{\mathrm{B}}}{2}+\tau g_{\text {orb }} \mu_{\mathrm{B}}\right) B_{\|}
$$

The dominant term is here the orbital contribution $[20,45,46]$. This can be clearly observed, e.g., in Fig. 5, where at large field the two visible conductance resonances correspond to two single-particle states with equal orbital angular moment but opposite spin. As a consequence, we go one step further and classify our states only by orbital angular momentum direction (corresponding to $\mathrm{K}^{\prime}$ or $\mathrm{K}$ valley), i.e. by dominant magnetic-field dispersion:

$$
E_{\tau}\left(B_{\|}\right)=\tau g_{\text {orb }} \mu_{\mathrm{B}} B_{\|}, \quad \tau= \pm 1 .
$$

In the case of the second SET region where the charge occupation is $1 \leqslant N_{\mathrm{el}} \leqslant 2$, resonance lines correspond to energy differences $\Delta E_{12}\left(B_{\|}\right)=E_{2}\left(B_{\|}\right)-E_{1}\left(B_{\|}\right)$, and the dispersions in a magnetic field, correspondingly, to the orbital angular momentum change when a second electron tunnels onto the quantum dot, proportional to

$$
\frac{\mathrm{d}\left(\Delta E_{12}\left(B_{\|}\right)\right)}{\mathrm{d} B_{\|}}=\frac{\mathrm{d}\left(E_{2}\left(B_{\|}\right)-E_{1}\left(B_{\|}\right)\right)}{\mathrm{d} B_{\|}} .
$$

If the angular momentum of the first electron remains unchanged when the second eletron tunnels onto the dot, only two slopes corresponding to addition of a $\mathrm{K}^{\prime}$ or $\mathrm{K}$ electron remain possible. From this we can classify the conductance lines of Fig. 3(a). In the low-bias region of the figure, two dominant slopes clearly emerge; we identify these with the addition of a $\mathrm{K}^{\prime}$-valley electron (downward slope) or a K-valley electron (upward slope), respectively $[20,45,46]$.

\section{APPENDIX D: EVALUATING THE SIDEBANDS OF EXCITED STATES}

The data points of Fig. 4 are obtained as follows. We separate the conductance resonance pattern into line segments, each terminated by line crossings or anticrossings. On each segment that conforms to one of the two dominant slopes, we select a point $\left(B_{\|}, V_{\mathrm{sd}}\right)$ near the high- $B_{\|}$end of the segment; these points are the locations of the symbols in Fig. 4(a). The five traces $G\left(V_{\text {sd }}\right)$ closest to this point in magnetic field are evaluated using the fit procedure as described above. To reduce the impact of outliers, the plotted values in Fig. 4 are then given by the median of the fit parameters $g$ from these five evaluations.

\section{APPENDIX E: VARIABLE COUPLING MODEL}

One of the key results of Ref. [20], based on Refs. [50-52], is that a magnetic field parallel to the axis of a carbon nanotube does not only modify the circumferential electronic wave function via an Aharonov-Bohm phase, but also the wave function in axial direction. While a typical onedimensional quantum box, i.e., a potential well with hard walls of infinite height, forces the wave function of a trapped particle to zero at both ends, this construction is not possible for carbon nanotubes. With the exception of armchair nanotubes, there are no solutions that allow the electronic wave function to become zero on both ends for both graphene sublattices. Instead, the longitudinal quantization can be given by the condition that the wave function on each sublattice becomes zero at the end where that particular sublattice provides the majority of boundary atoms. With $L$ as the length of the nanotube segment, this introduces a so-called crossquantization condition [20]:

$$
e^{2 i \kappa_{\|} L} \stackrel{!}{=} \frac{\tau \kappa_{\perp}+i \kappa_{\|}}{\tau \kappa_{\perp}-i \kappa_{\|}}
$$



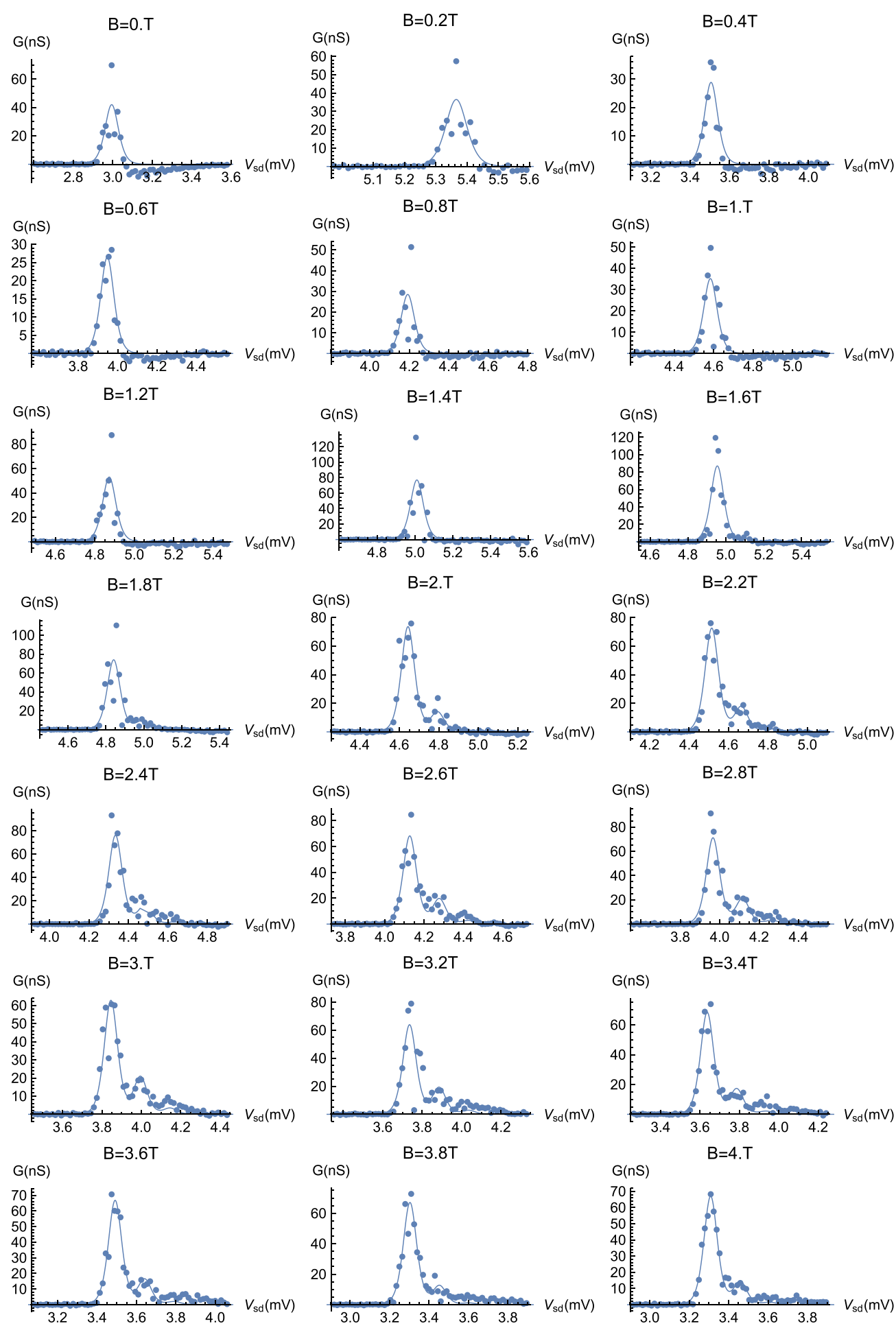

FIG. 10. Example plots of numerically obtained differential conductance corresponding to data traces of Fig. 2(b) and the fits resulting in the parameters plotted in Fig. 2(f) (blue squares there).

The axial wave number $\kappa_{\|}$and the circumferential wave number $\kappa_{\perp}$ of an electronic state are coupled; one example solution is sketched in Fig. 6(a) following Ref. [20]. The axial magnetic field tunes the longitudinal profile and charge distribution of the electronic wave function from a $\lambda / 4$ resonator like shape, with a finite value at one edge, near $B_{\|}=0$ all the way to a $\lambda / 2$ resonator like shape at high field; see the schematic drawings in Fig. 6(a).

In Ref. [20], wave-function envelopes have been calculated numerically using a tight-binding approach. Here, we approximate these results using a simple functional dependence: for 

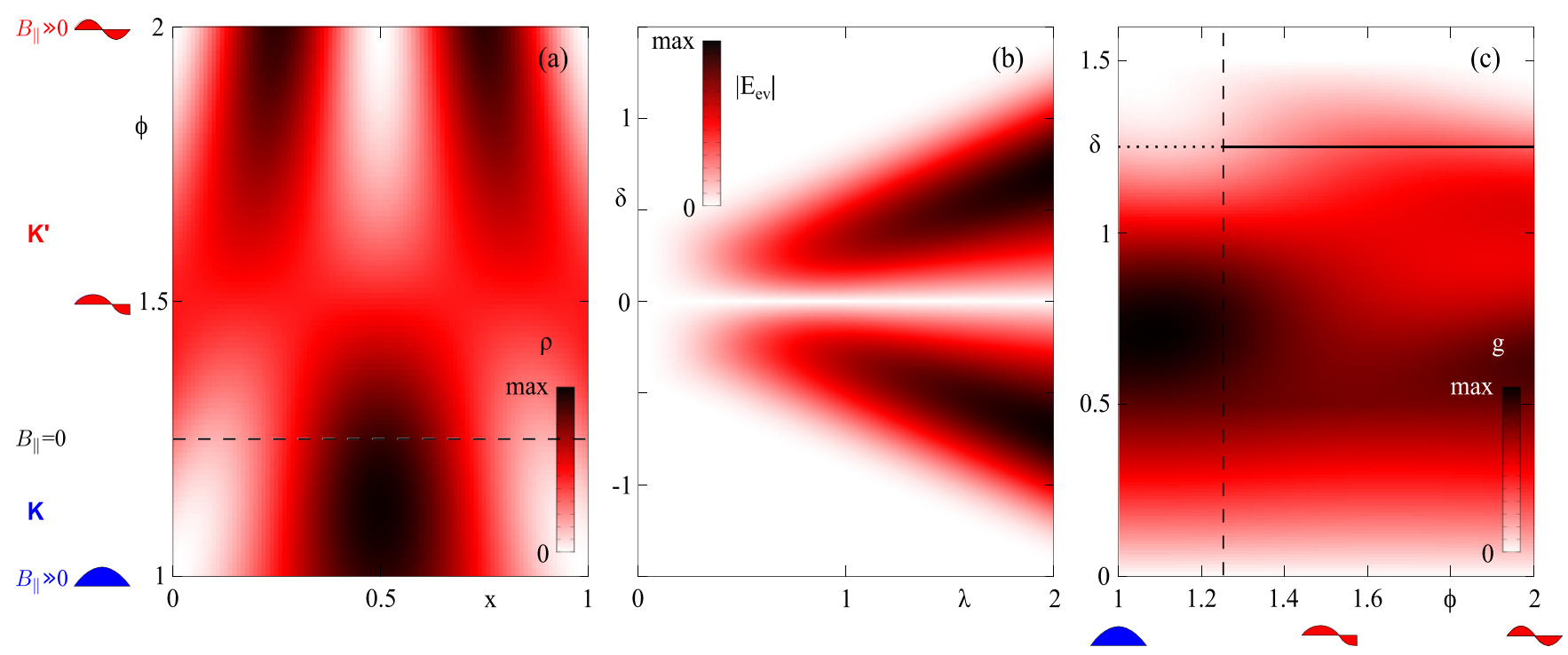

FIG. 11. Model construction: (a) Assumed linear single-particle charge density $\rho$ along a nanotube quantum dot of length 1, as function of position $x$ and number of antinodes of the graphene sublattice wave function $\phi$, cf. [20]. The approximate situation at low magnetic field is marked with a dashed line at $\phi=1.25 ; \phi=2$ corresponds to the high field limit of the $\mathrm{K}^{\prime}$ state, $\phi=1$ to the high field limit of the corresponding K state. (b) Absolute value of the interaction energy $\left|E_{\mathrm{ev}}\right|$ for $\phi=1$, as function of length ratio $\lambda=L_{v} / L_{d}$ and relative position of the centers $\delta=\left(x_{v}-x_{d}\right) / L_{d}$ of vibron and quantum dot. (c) Franck-Condon parameter $g$, approximated as $g \simeq E_{\mathrm{ev}}^{2}$, for fixed $\lambda=2$ and as function of $\phi$ and $\delta$. The solid/dotted line at $\delta=1.25$ corresponds to the line cuts shown in Fig. 6(c); $\phi=1.25$ is again marked with a dashed line.

a quantum dot of length 1 , we write for sublattice A

$$
\psi_{A}(x, \phi)=\sin (\pi \phi x), \quad x \in[0,1]
$$

and, accordingly, for sublattice B

$$
\psi_{B}(x, \phi)=\psi_{A}(1-x, \phi) .
$$

The parameter $\phi$ describes the number of wave-function antinodes along the quantum dot, i.e., in the range $x \in$ $[0,1]$. Half-wavelength resonator solutions are given by $\phi=$ $1,2, \ldots$, quarter-wavelength resonator solutions are given by $\phi=0.5,1.5,2.5, \ldots$ As can be seen in Fig. 6(a), increasing a magnetic field from zero continuously tunes $\phi$, for a $\mathrm{K}^{\prime}$ state from $\phi \approx 1.25$ toward $\phi=2$, for a $\mathrm{K}$ state from $\phi \approx 1.25$ toward $\phi=1$.

To obtain the total linear electron density, we now sum up the densities on the two sublattices, while normalizing such that a change in $\phi$ does not modify the total charge:

$$
\rho(x, \phi)=\frac{\left|\psi_{A}(x, \phi)\right|^{2}+\left|\psi_{B}(x, \phi)\right|^{2}}{\int_{0}^{1}\left|\psi_{A}(x, \phi)\right|^{2}+\left|\psi_{B}(x, \phi)\right|^{2} \mathrm{~d} x} .
$$

The result is plotted in Fig. 11(a).

Reference [37] discusses the electron-vibron interaction of an interacting electron system using a Tomonaga-Luttinger model. From the interaction Hamiltonian used there, Eq. (12) in Ref. [37], coupling the electron density to the deformation potential, we take inspiration of our interaction energy,

$$
\left.E_{\mathrm{ev}}(\phi) \propto \int \rho(x, \phi)\right) \frac{\mathrm{d} u}{\mathrm{~d} x} \mathrm{~d} x
$$

where $u(x)$ is the vibration envelope of the mechanical mode. In addition, also following Ref. [37] and its nomenclature, we allow for the vibron and the quantum dot to be shifted relative to each other and to be of different lengths; we parametrize this using length ratio $\lambda=L_{v} / L_{d}$ and relative position of the centers $\delta=\left(x_{v}-x_{d}\right) / L_{d}$ of vibron and quantum dot. Fig. 11(b) plots for $\phi=1$ the interaction energy $\left|E_{\mathrm{ev}}(\lambda, \delta)\right|$, reproducing basic features of the corresponding TomonagaLuttinger model plot, left panel of Fig. 5 in Ref. [37].

Given the comparatively small vibrational energy quantum in our device, cf. Fig. 8, and the strong electrostatic confinement of the quantum dot, we consider it likely that the quantum dot is smaller than the vibron envelope, i.e., $\lambda>1$. We are now interested in the magnetic-field-induced modification of the Franck-Condon parameter $g \propto E_{\mathrm{ev}}^{2}$ [37] and thus plot in Fig. 11(c) this value as function of $\phi$ and $\delta$, for a fixed $\lambda=2$. The solid/dotted line corresponds to $\delta=1.25$, the value selected for the trace cut in Fig. 6(c).
[1] J. Franck, Elementary processes of photochemical reactions, Trans. Faraday Soc. 21, 536 (1926).

[2] E. Condon, A theory of intensity distribution in band systems, Phys. Rev. 28, 1182 (1926).
[3] H. Park, J. Park, A. K. L. Lim, E. H. Anderson, A. P. Alivisatos, and P. L. McEuen, Nanomechanical oscillations in a single-C60 transistor, Nature 407, 57 (2000). 
[4] S. Braig and K. Flensberg, Vibrational sidebands and dissipative tunneling in molecular transistors, Phys. Rev. B 68, 205324 (2003).

[5] B. J. LeRoy, S. G. Lemay, J. Kong, and C. Dekker, Electrical generation and absorption of phonons in carbon nanotubes, Nature 432, 371 (2004).

[6] E. M. Weig, R. H. Blick, T. Brandes, J. Kirschbaum, W. Wegscheider, M. Bichler, and J. P. Kotthaus, Single-ElectronPhonon Interaction in a Suspended Quantum Dot Phonon Cavity, Phys. Rev. Lett. 92, 046804 (2004).

[7] J. Koch and F. von Oppen, Franck-Condon Blockade and Giant Fano Factors in Transport Through Single Molecules, Phys. Rev. Lett. 94, 206804 (2005).

[8] W. Izumida and M. Grifoni, Phonon-assisted tunneling in interacting suspended single-wall carbon nanotubes, New J. Phys. 7, 244 (2005).

[9] S. Sapmaz, P. Jarillo-Herrero, Ya. M. Blanter, C. Dekker, and H. S. J. van der Zant, Tunneling in Suspended Carbon Nanotubes Assisted by Longitudinal Phonons, Phys. Rev. Lett. 96, 026801 (2006).

[10] J. Koch, F. von Oppen, and A. V. Andreev, Theory of the Franck-Condon blockade regime, Phys. Rev. B 74, 205438 (2006)

[11] E. A. Osorio, K. O’Neill, N. Stuhr-Hansen, O. F. Nielsen, T. Bjørnholm, and H. S. J. van der Zant, Addition energies and vibrational fine structure measured in electromigrated single-molecule junctions based on an oligophenylenevinylene derivative, Adv. Mater. 19, 281 (2007).

[12] E. Burzurí, Y. Yamamoto, M. Warnock, X. Zhong, K. Park, A. Cornia, and H. S. J. van der Zant, Franck-Condon blockade in a single-molecule transistor, Nano Lett. 14, 3191 (2014).

[13] A. K. Hüttel, M. Poot, B. Witkamp, and H. S. J. van der Zant, Nanoelectromechanics of suspended carbon nanotubes, New J. Phys. 10, 095003 (2008).

[14] R. Leturcq, C. Stampfer, K. Inderbitzin, L. Durrer, C. Hierold, E. Mariani, M. G. Schultz, F. von Oppen, and K. Ensslin, Franck-Condon blockade in suspended carbon nanotube quantum dots, Nat. Phys. 5, 327 (2009).

[15] A. K. Hüttel, B. Witkamp, M. Leijnse, M. R. Wegewijs, and H. S. J. van der Zant, Pumping of Vibrational Excitations in the Coulomb-Blockade Regime in a Suspended Carbon Nanotube, Phys. Rev. Lett. 102, 225501 (2009).

[16] J. O. Island, V. Tayari, A. C. McRae, and A. R. Champagne, Few-hundred $\mathrm{GHz}$ carbon nanotube nanoelectromechanical systems (NEMS), Nano Lett. 12, 4564 (2012).

[17] M. Ganzhorn, S. Klyatskaya, M. Ruben, and W. Wernsdorfer, Strong spin-phonon coupling between a single-molecule magnet and a carbon nanotube nanoelectromechanical system, Nat. Nanotechnol. 8, 165 (2013).

[18] M. Jung, J. Schindele, S. Nau, M. Weiss, A. Baumgartner, and C. Schönenberger, Ultraclean single, double, and triple carbon nanotube quantum dots with recessed Re bottom gates, Nano Lett. 13, 4522 (2013).

[19] P. Weber, H. L. Calvo, J. Bohle, K. Goß, C. Meyer, M. R. Wegewijs, and C. Stampfer, Switchable coupling of vibrations to two-electron carbon-nanotube quantum dot states, Nano Lett. 15, 4417 (2015).

[20] M. Margańska, D. R. Schmid, A. Dirnaichner, P. L. Stiller, Ch. Strunk, M. Grifoni, and A. K. Hüttel, Shaping Electron Wave
Functions in a Carbon Nanotube with a Parallel Magnetic Field, Phys. Rev. Lett. 122, 086802 (2019).

[21] J. Cao, Q. Wang, and H. Dai, Electron transport in very clean, as-grown suspended carbon nanotubes, Nat. Mater. 4, 745 (2005).

[22] K. J. G. Götz, D. R. Schmid, F. J. Schupp, P. L. Stiller, Ch. Strunk, and A. K. Hüttel, Nanomechanical Characterization of the Kondo Charge Dynamics in a Carbon Nanotube, Phys. Rev. Lett. 120, 246802 (2018).

[23] D. R. Schmid, P. L. Stiller, A. Dirnaichner, and A. K. Hüttel, From Fabry-Pérot interference to Coulomb blockade at fixed hole number, arXiv:2005.01183 (2020).

[24] J. Park and P. L. McEuen, Formation of a p-type quantum dot at the end of an n-type carbon nanotube, Appl. Phys. Lett. 79, 1363 (2001).

[25] G. A. Steele, G. Gotz, and L. P. Kouwenhoven, Tunable fewelectron double quantum dots and Klein tunneling in ultraclean carbon nanotubes, Nat. Nanotechnol. 4, 363 (2009).

[26] At higher fields $B_{\|} \gtrsim 6 \mathrm{~T}$ the extracted peak positions may indicate an increase in oscillator quantum, though the signal/noise ratio makes a quantitative analysis challenging here.

[27] A. Zazunov, D. Feinberg, and T. Martin, Phonon-mediated negative differential conductance in molecular quantum dots, Phys. Rev. B 73, 115405 (2006).

[28] R. Härtle, C. Benesch, and M. Thoss, Vibrational Nonequilibrium Effects in the Conductance of Single Molecules with Multiple Electronic States, Phys. Rev. Lett. 102, 146801 (2009).

[29] F. von Oppen, F. Guinea, and E. Mariani, Synthetic electric fields and phonon damping in carbon nanotubes and graphene, Phys. Rev. B 80, 075420 (2009).

[30] E. Mariani and F. von Oppen, Electron-vibron coupling in suspended carbon nanotube quantum dots, Phys. Rev. B 80, 155411 (2009).

[31] H. Hübener and T. Brandes, Master equations for quantum transport with Franck-Condon blockade, Phys. Rev. B 80, 155437 (2009).

[32] F. Cavaliere, E. Mariani, R. Leturcq, C. Stampfer, and M. Sassetti, Asymmetric Franck-Condon factors in suspended carbon nanotube quantum dots, Phys. Rev. B 81, 201303(R) (2010).

[33] S. Maier, T. L. Schmidt, and A. Komnik, Charge transfer statistics of a molecular quantum dot with strong electron-phonon interaction, Phys. Rev. B 83, 085401 (2011).

[34] R. Härtle and M. Thoss, Vibrational instabilities in resonant electron transport through single-molecule junctions, Phys. Rev. B 83, 125419 (2011).

[35] A. Yar, A. Donarini, S. Koller, and M. Grifoni, Dynamical symmetry breaking in vibration-assisted transport through nanostructures, Phys. Rev. B 84, 115432 (2011).

[36] T.-F. Fang, Q.-F. Sun, and H.-G. Luo, Phonon-assisted transport through suspended carbon nanotube quantum dots, Phys. Rev. B 84, 155417 (2011)

[37] A. Donarini, A. Yar, and M. Grifoni, Spectrum and FranckCondon factors of interacting suspended single-wall carbon nanotubes, New J. Phys. 14, 023045 (2012).

[38] S.-N. Zhang, W. Pei, T.-F. Fang, and Q.-F. Sun, Phonon-assisted transport through quantum dots with normal and superconducting leads, Phys. Rev. B 86, 104513 (2012). 
[39] A. Jovchev and F. B. Anders, Influence of vibrational modes on quantum transport through a nanodevice, Phys. Rev. B 87, 195112 (2013).

[40] L. Simine and D. Segal, Electron transport in nanoscale junctions with local anharmonic modes, J. Chem. Phys. 141, 014704 (2014).

[41] A. McCaskey, Y. Yamamoto, M. Warnock, E. Burzurí, H. S. J. van der Zant, and K. Park, Electron-vibron coupling effects on electron transport via a single-molecule magnet, Phys. Rev. B 91, 125419 (2015).

[42] J. K. Sowa, J. A. Mol, G. A. D. Briggs, and E. M. Gauger, Vibrational effects in charge transport through a molecular double quantum dot, Phys. Rev. B 95, 085423 (2017).

[43] C. W. J. Beenakker, Theory of Coulomb-blockade oscillations in the conductance of a quantum dot, Phys. Rev. B 44, 1646 (1991).

[44] M. Margańska (private communication).

[45] E. D. Minot, Y. Yaish, V. Sazonova, and P. L. McEuen, Determination of electron orbital magnetic moments in carbon nanotubes, Nature 428, 536 (2004).

[46] E. A. Laird, F. Kuemmeth, G. A. Steele, K. Grove-Rasmussen, J. Nygård, K. Flensberg, and L. P. Kouwenhoven, Quantum transport in carbon nanotubes, Rev. Mod. Phys. 87, 703 (2015).

[47] C. Enss and S. Hunklinger, Low-Temperature Physics (Springer-Verlag, Berlin, Heidelberg, New York, 2005).

[48] D. R. Schmid, P. L. Stiller, Ch. Strunk, and A. K. Hüttel, Liquid-induced damping of mechanical feedback effects in single electron tunneling through a suspended carbon nanotube, Appl. Phys. Lett. 107, 123110 (2015).

[49] D. R. Schmid, S. Smirnov, M. Marganska, A. Dirnaichner, P. L. Stiller, M. Grifoni, A. K. Hüttel, and C. Strunk, Broken SU(4) symmetry in a Kondo-correlated carbon nanotube, Phys. Rev. B 91, 155435 (2015).

[50] A. R. Akhmerov and C. W. J. Beenakker, Boundary conditions for Dirac fermions on a terminated honeycomb lattice, Phys. Rev. B 77, 085423 (2008).

[51] A. H. Castro Neto, F. Guinea, N. M. R. Peres, K. S. Novoselov, and A. K. Geim, The electronic properties of graphene, Rev. Mod. Phys. 81, 109 (2009).

[52] M. Marganska, M. del Valle, S. H. Jhang, C. Strunk, and M. Grifoni, Localization induced by magnetic fields in carbon nanotubes, Phys. Rev. B 83, 193407 (2011).

[53] A. Benyamini, A. Hamo, S. Viola Kusminskiy, F. von Oppen, and S. Ilani, Real-space tailoring of the electron-phonon coupling in ultraclean nanotube mechanical resonators, Nat. Phys. 10, 151 (2014).

[54] S. Reinhardt, C. Butschkow, S. Geissler, A. Dirnaichner, F. Olbrich, C. Lane, D. Schröer, and A. K. Hüttel, Lab::Measurement-a portable and extensible framework for controlling laboratory equipment and conducting measurements, Comput. Phys. Commun. 234, 216 (2019).

[55] D. R. Schmid, P. L. Stiller, C. Strunk, and A. K. Hüttel, Magnetic damping of a carbon nanotube nano-electromechanical resonator, New J. Phys. 14, 083024 (2012). 